\title{
Evaluation of commercial serological assays in Malaysia for detection of anti-Zika virus antibodies
}

\author{
Khoo, H.Y. ${ }^{1}$, Lee, H.Y. ${ }^{1 *}$, Khor, C.S. ${ }^{1}$, Tan, K.K. ${ }^{1}$, AbuBakar, S. ${ }^{1,2,3^{*}}$ \\ ${ }^{1}$ Tropical Infectious Diseases Research \& Education Centre (TIDREC), Higher Institution Centre of Excellence (HICoE), Universiti Malaya, \\ 50603 Kuala Lumpur, Malaysia \\ ${ }^{2}$ WHO Collaboration Centre for Arbovirus Reference and Research Malaysia, Universiti Malaya, 50603 Kuala Lumpur, Malaysia (MAA-12) \\ ${ }^{3}$ Department of Medical Microbiology, Faculty of Medicine, Universiti Malaya, 50603 Kuala Lumpur, Malaysia \\ *Corresponding author: sazaly@um.edu.my; leehaiyen@um.edu.my
}

\section{ARTICLE HISTORY}

Received: 20 October 2021

Revised: 14 December 2021

Accepted: 14 December 2021

Published: 31 December 2021

\begin{abstract}
The recommended test guidelines for Zika virus (ZIKV) include using both molecular and serological tools. While the molecular tools are useful for detecting acute infection, the serological tools are useful for the detection of previous infections. Nevertheless, detection of ZIKV-specific antibodies remains a challenge due to the high cross-reactivity between ZIKV and other flaviviruses such as dengue virus (DENV) and Japanese encephalitis virus (JEV). The objective of this study is to evaluate the commercially available enzyme-linked immunosorbent assay (ELISA) for the detection of ZIKV IgG. In this study, we evaluated 6 commercially available anti-ZIKV IgG ELISA kits. Pre-characterized serum panels consisting of 70 sera were selected for the evaluation. The diagnostic accuracy of each ELISA kits was determined and compared to the gold standard, Foci Reduction Neutralization Test (FRNT). The present study established that the performance of the NS1-based anti-ZIKV IgG ELISA kit was superior to that which uses of the E protein as antigen. Overall, commercial ZIKV IgG ELISA showed varying test performances, with some achieving moderate to high test sensitivities and specificities. When compared against the FRNT, the test sensitivities ranged from $7.1 \%$ to $78.6 \%$, whereas, the test specificities ranged from $40.0 \%$ to $100 \%$. Limitation to the study includes the cross reactivity between flavivirus and specificity of the kit in addressing the cross reactivity.
\end{abstract}

Keywords: Infectious diseases; vector-borne diseases; antibodies; Zika virus; ELISA.

\section{INTRODUCTION}

Zika virus (ZIKV) is an emerging mosquito-borne virus that belongs to the Flavivirus genus and in the Flaviviridae family. There are other medically important flaviviruses which include dengue virus (DENV), Japanese encephalitis virus (JEV), West Nile virus (WNV), and yellow fever virus (YFV) (Dick et al., 1952). The virus was first isolated from a captive rhesus macaque (Macaca mulatta) from the Zika Forest, Uganda in 1947 (Dick et al., 1952). A single blood meal by infected Aedes species mosquitoes (e.g., Ae. aegypti and Ae. albopictus) is enough to infect human with the virus (GutiérrezBugallo et al., 2019). The spread of mosquito vectors and the viruses they carry are facilitated by human mobility and travel (Musso \& Gubler, 2016). The virus presence is well established around the world, especially in the Pacific Islands and the Americas (Musso et al., 2019). The autochthonous transmission of ZIKV has been reported in over 87 countries and territories (WHO, 2019).

The current guidelines by the United States Centers for Disease Control and Prevention (CDC) and the World Health
Organization (WHO) for the laboratory diagnosis of Zika virus infection is limited to a positive reverse-transcription polymerase chain reaction (RT-PCR) within 2 weeks of postsymptoms onset for confirmation of Zika virus infection, and a negative dengue-lgM test to exclude dengue virus infection (Rabe et al., 2016; WHO, 2016a). Moreover, a positive IgM tests need to be confirmed by a more specific neutralization test only when IgM test shows positive to both DENV and ZIKV. Detection of ZIKV RNA in the laboratory diagnosis is highly specific but limited to the short detection window (within one week after symptoms onset) (Fourcade et al., 2016). Both neutralizing (primarily IgG class) and non-neutralizing antiZIKV antibodies are produced during infection. Anti-ZIKV IgM antibodies are detectable from the first week to 4 weeks of post-infection, whereas, anti-ZIKV IgG antibodies remain detectable from 4 weeks onward post-infection (Landry \& St George, 2017). Generally, anti-ZIKV IgM and non-specific antibodies both decline after several months, but anti-ZIKV IgG can be detected for many months and may persist for decades (Landry \& St George, 2017). Meanwhile, an IgG test is not routinely recommended. 
Most antibodies elicited by flavivirus infection recognize the structural envelope (E) protein and the nonstructural protein 1 (NS1). As the $E$ protein contains the major neutralizing epitopes, serological tests for flaviviruses have previously focused on the E protein (Dai et al., 2016). However, due to the extensive cross-reactivity of ZIKV with other flaviviruses, ZIKV NS1 has been used as the alternative to $E$ protein in many studies (Saba Villarroel et al., 2018; Langerak et al., 2019; Nguyen et al., 2020). ZIKV NS1 is highly conserved and structurally similar with other flavivirus NS1 protein (Song et al., 2016). Its unique surface electrostatic potential could alter the binding properties to known protective (neutralizing) antibodies to other flavivirus NS1 protein, giving a specific NS1 surface characteristic among other flaviviruses (Song et al., 2016). However, the cross-reactivity of anti-NS1 antibodies in patients with previous dengue infections cannot be fully excluded. In addition, DENV and Chikungunya virus (CHIKV) are co-circulating in overlapping regions with ZIKV, and all are being transmitted by the same mosquito species (Wilder-Smith et al., 2018). Although CHIKV is an Alphavirus, serological cross-reactivity in acute dengue cases was reported (Lima et al., 2021). Therefore, a commercially available serological assay with good reliability is urgently needed to enable surveillance study to be undertaken commercially in determining the extent of ZIKV prevalence and its potential risk to the population. This is especially important for the development of a Zika virus vaccine where its potential use is important in regions where Zika virus infection is endemic.

Currently, a large number of kits for the detection of antibodies against ZIKV are commercially available (Ohst et al., 2018). However, the utility of ELISA kits in ZIKV seroprevalence studies remains a challenge due to the high cross-reactivity between flaviviruses. A comparative evaluation of a selection of these kits is of interest for the screening of antibodies against Zika virus infection. Therefore, the objective of this study is to evaluate the performance characteristics of six commercially available enzyme-linked immunosorbent assay (ELISA) kits for their potential use in Malaysia.

\section{MATERIALS AND METHODS}

This study was conducted in compliance with the Standards for Reporting of Diagnostic Accuracy (STARD) guidelines (Bossuyt et al., 2015).

\section{Approval}

The Institutional Review Board (IRB) approval for the study was obtained from the University of Malaya Research Ethics Committee (UM.TNC2/UMREC-680) for the access to the archived serum samples.

\section{Selection of pre-characterized human serum panels}

The present study used the archived serum samples received from Tropical Infectious Diseases Research and Education Centre (TIDREC). These samples were screened for IgG antibodies screening using anti-ZIKV IgG ELISA kits. All samples were stored at $-80^{\circ} \mathrm{C}$ after routine diagnostic testing until included in this study for evaluation. The inclusion criteria for the sample selection were presence of data for all the diagnostics tests involved and sufficient sample volume. A total of 70 of the 621 archived serum samples met the inclusion criteria and were selected as the serum panels for this study. The serum panels were classified as ZIKV subgroup and non-ZIKV subgroup, as shown in Table 1. ZIKV subgroup consisted of 10 serum samples (designated as
ZIKV+) previously characterized positive for ZIKV IgG only but negative for DENV NS1, DENV IgM, DENV IgG, JEV IgG, and CHIKV IgG. Non-ZIKV subgroup consisted of 60 serum samples that were negative for ZIKV IgG. Among these, there were 12 samples positive for DENV IgG (designated as DENV+); 12 samples positive for JEV IgG (designated as JEV+); 12 samples positive for CHIKV IgG (designated as CHIKV+); and 24 samples absence of IgG for all the viruses (designated as negative controls).

\section{Selection of anti-ZIKV IgG ELISA kits}

A total of six readily available anti-ZIKV IgG ELISA kits were evaluated. These six commercially available anti-ZIKV IgG ELISA kits: Brand A ELISA kit (UK), Brand B ELISA kit (USA), Brand C ELISA kit (Germany), Brand D ELISA kit (USA), Brand E ELISA kit (USA), and Brand F ELISA kit (USA). The basic features of the ELISA kits were summarized (Supplementary Table S1, available upon request). Brand C ELISA kit used recombinant NS1 protein as the antigen. All the remaining NS1-based ZIKV ELISA kits (Brand A, B, D, E) used an unspecified Zika virus antigen, whereas only the Brand F ELISA kit used recombinant $E$ as the antigen. The selection of kits was based on their availability of the ELISA kits in the market in Malaysia.

\section{Enzyme-linked immunosorbent assays}

All the ELISA kits were assessed based on the accuracy of results and comparative features of each kit. Kits were received in good condition and were stored at the manufacturers' recommended storage condition. Results were classified according to the instructions of the individual kit and repeat testing of samples with equivocal results were not performed. All samples were tested using each kit strictly based on the manufacturer's specifications. Supplementary Table 1 summarizes the sample dilution, antigen type coated on ELISA kits, and interpretation of the cut-off value of the test results. Serum samples with ZIKV seropositive on ELISA but was from the non-ZIKV subgroup panel were further validated using FRNT assay.

\section{Foci reduction neutralization test}

The FRNT assay was implemented as previously described (Sam et al., 2019; Khor et al., 2020) with slight modification using mouse anti-ZIKV NS1 monoclonal antibodies (Abcam, UK) and HRP antibody (Abcam, UK) as secondary antibodies to stain foci formed by infected Vero cells (CCL-81). FRNT 90 was performed to validate the ELISA results and address potential bias to any commercially available ELISA kits. Neutralization was defined as the serum dilution that resulted in $90 \%$ reduction in the number of virus-induced foci $\left(F_{R N T_{90}}\right.$ ) as compared to control wells (virus control and negative control). Serum dilution was performed at the final dilution of 1:40 after adding an equivalent volume of virus (200 ul). PRNT PR $_{90}$ titers were recommended by WHO for flavivirus PRNT application to improve specificity, by decreasing the background serum cross-reactivities among flaviviruses (Roehrig et al., 2008; WHO, 2016b). PRNT which was recommended as reference standard for ZIKV as previously described was subsequently substituted by FRNT (Rabe et al., 2016). This is because FRNT method stained the ZIKV infected cells directly, whereas, PRNT depended on the plaque formation which might also be affected by the noninfected cells that being fell off (Vaidya et al., 2010).

\section{Statistical analysis}

Statistical analysis was performed using the IBM SPSS version 25.0 (IBM Corp., Armonk, NY). Diagnostic accuracy and 
agreement rates were calculated together with sensitivity, specificity, positive predictive value (PPV), and negative predictive value (NPV) for each kit. Receiver operating characteristic (ROC) was also performed and the area under the ROC curve $(A \cup C)$ that measured how well predictions were ranked, and were used for the whole picture depiction of the accuracy of each kit. In particular AUC of $>0.89,0.50$ to 0.89 , and $<0.50$ were indicative of excellent, moderate, and poor in differentiating ZIKV-infected serum sample from a non-ZIKV-infected serum sample, respectively based on FRNT results (Hajian-Tilaki, 2013).

\section{RESULTS}

In this study, a two-stage evaluation of ELISA kits was implemented. This comprised an evaluation using the developmental serum panels in Phase I and a validation panel in Phase II (Table 1). The developmental serum panels in Phase I $(n=70)$ comprised serum panel pre-characterized by ELISAs for all the viruses, whereas, validation serum samples in Phase II $(n=19)$ sought confirmation by ZIKV FRNT. An algorithm for ZIKV serostatus clarification using anti-ZIKV NS1 IgG ELISA, anti-DENV NS1 IgG ELISA, and FRNT 90 was presented in Figure 1.

\section{Evaluation of anti-ZIKV IgG ELISA kits}

Results from all the anti-ZIKV IgG ELISA kits were examined to determine their sensitivity and specificity using the developmental serum panel $(n=70)$. Sixty-nine percent $(48 /$ 70) of the samples were negative in all the ELISAs, while no sample was positive in all the ELISAs. The remaining $31 \%$ $(22 / 70)$ of the samples were positive in at least one of the ELISAs. As shown in Table 2, in the ZIKV+ samples, the NS1based ELISA sensitivity ranged from $10 \%-100 \%$. Sensitivity for the Brand C ELISA kit was $100 \%$, followed by Brand A ELISA kit with $20 \%$. The Brand B, Brand D, and Brand E ELISA kits showed only $10 \%$ sensitivity.

Assay specificity was assessed by testing against 36 cross-reactive samples and 24 negative controls. The NS1based ELISA specificity ranged from $93.3 \%$ to $100 \%$, where $2 /$ 12 (16.7\%, CHIKV), 3/12 (25\%, DENV), and 2/24 (8.3\%, negative controls) samples were found to be anti-ZIKV IgG positive, while the remaining were negative. Both false-positive samples from the negative controls were identified from the Brand D and Brand E ELISA kits.

Although the Brand B ELISA kit showed a specificity of $100 \%$, it has low sensitivity (sensitivity $=10 \%$ ) as it identified $90 \%(n=9)$ of ZIKV+ samples as negative. Specificity of the Brand A ELISA kit was $95 \%$ followed by $93.3 \%$ for Brand C, Brand D, and Brand E ELISA kits. False positives reported from these ELISA kits were $5 \%(n=3)$ to $6.7 \%(n=4)$, respectively. Overall, false-negative results were high in all the ELISA kits when the results were at the borderline (equivocal) reading except for the Brand C ELISA kit.

Cross-reactivity was also analyzed from the 36 potentially cross-reactive samples. The Brand A ELISA kit's false-positive results were observed in DENV+ $(n=1 / 12)$, and CHIKV+ $(n=2 / 12)$ samples. On the other hand, the falsepositive results observed in the Brand D ELISA kit were in DENV+ $(n=1 / 12), \operatorname{CHIKV}+(n=1 / 12)$, and negative controls $(n=2)$ 24). However, for the Brand C ELISA kit, the majority of the false-positive results were observed in DENV+ $(n=3 / 12)$ followed by JEV+ $(n=1 / 12)$ samples. Both the Brand $B$ and Brand E ELISA kits did not have false-positive results reported. In contrast, unlike all the aforementioned ELISA kits, the Brand F ELISA kit which used ZIKV E protein had all samples resulting as false-positives.

Table 1. Characteristics of serum panels used in the study

Phase I: Developmental Serum Panels Serum testing panel
Pre-Characterization with ELISAs

Positive (Virus)

Negative (Virus)

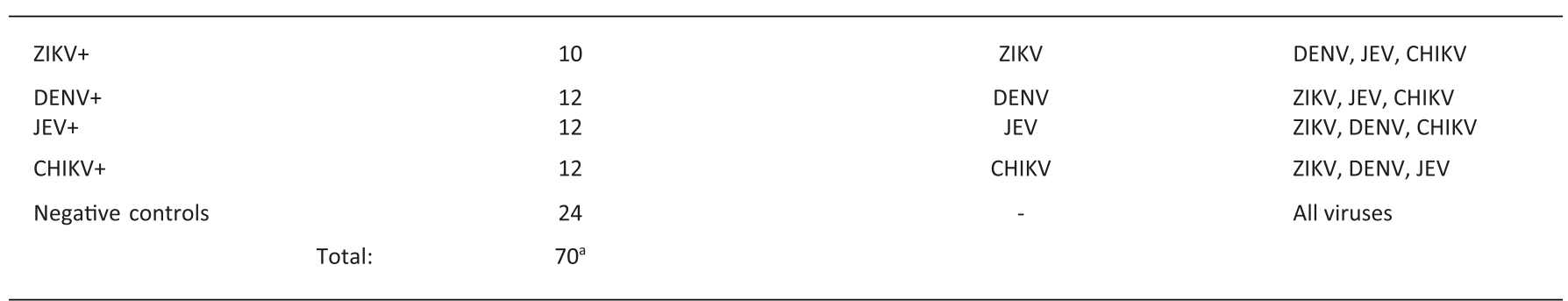

Phase II: Validation Serum Panels

Serum testing panel

FRNT $^{c}$

Pre-characterized

\begin{tabular}{l}
\multicolumn{1}{r}{ Pre-characterized No. of s } \\
ZIKV+ \\
DENV+ \\
JEV+ \\
CHIKV+ \\
Negative controls
\end{tabular}

Total: o. of specimens

Positive

Negative

8
6
1
3
1
$19^{b}$

$19^{b}$

8

4

1

1

0

14
0

2

0

2

1

5

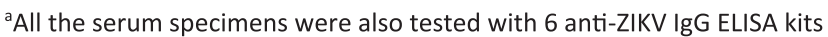

bSerum specimens showing mixed results from evaluated anti-ZIKV NS1 IgG ELISA kits

${ }^{\mathrm{c}} \mathrm{FRNT}$ as the reference standard test
} 


\section{Asym ptomatic people after exp osure to ZIKV in dengue endemic region}

4 weeks onward post infection to detect Ig

Ð serum

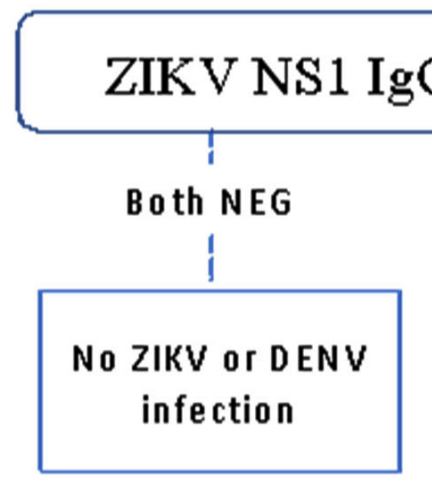

\section{and DENV NS1 IgG ELISAs}

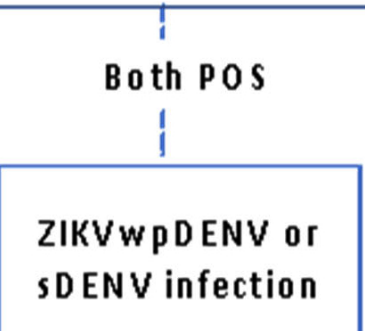

Either POS
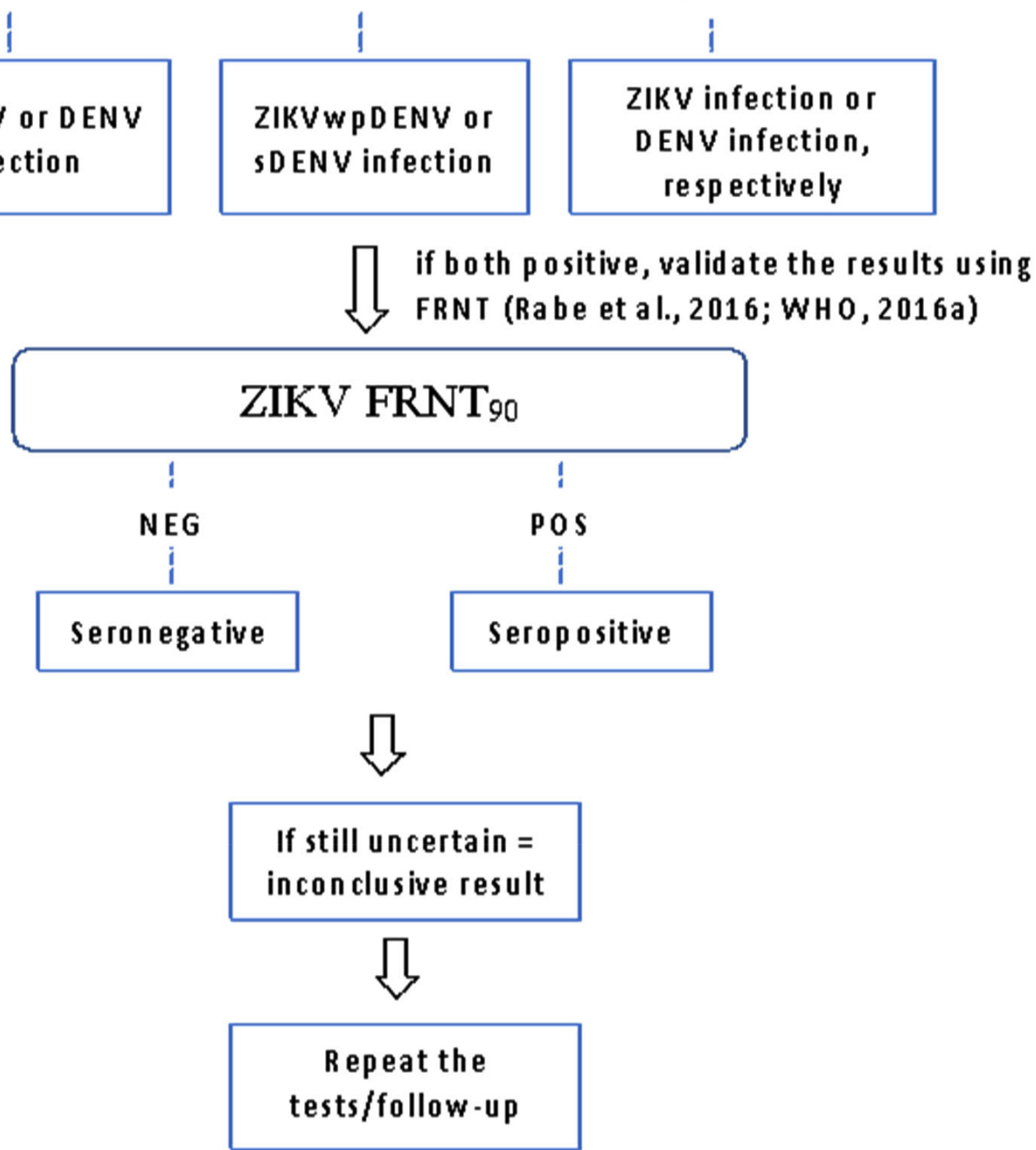

Figure 1. Algorithm for Zika serostatus classification using anti-ZIKV NS1 IgG ELISA, anti-DENV NS1 IgG ELISA, and FRNT 90 . ZIKVwpDENV infection: Zika virus infection with previous dengue virus infection. sDENV infection: secondary dengue virus infection.

Diagnostic accuracy of ELISA in comparison to FRNT

The diagnostic accuracy of each evaluated ELISA kit based on FRNT with the parameters: sensitivity, specificity, PPV positive predictive value, NPV - negative predictive value, AUC - area under the receiver operating characteristic curve analysis were presented in Table 3. The AUC analyse revealed overall moderate values $(0.271$ to 0.793$)$ for ZIKV IgG tests. Among the NS1-based assays only, the sensitivity observed from the Brand C ELISA kit was $78.6 \%$, with AUC $=0.793$ (95\% Cl: 0.549-1.000). The sensitivity of Brand D ELISA kit ranked second, which was $21.4 \%$, followed by the Abcam kit with $14.3 \%$. Both the Brand B and Brand E ELISA kit obtained low sensitivity of only $7.1 \%$. On the other hand, the highest specificity was obtained with the Brand B and Brand E ELISA kits which were at $100 \%$ respectively. Both the Brand $C$ and Brand D ELISA kits obtained a specificity of $80.0 \%$, respectively, 
Khoo et al. (2021), Tropical Biomedicine 38(4): 613-621

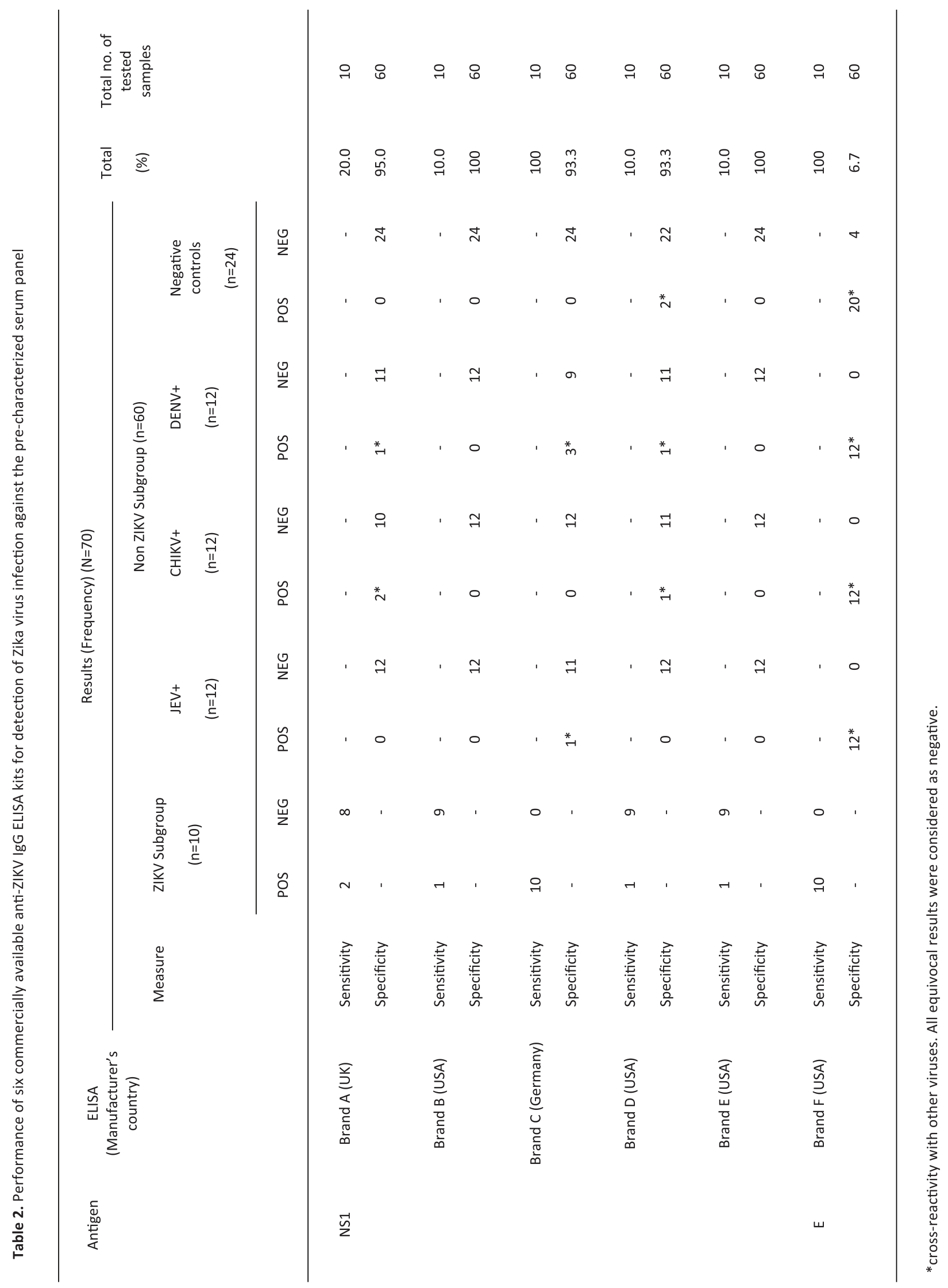


Table 3. Diagnostic accuracy and parameters of each ELISA kit compared against FRNT $90(n=19)$

\begin{tabular}{|c|c|c|c|c|c|c|}
\hline Antigen & ELISA & SS & SP & PPV & NPV & $\operatorname{AUC}(95 \% \mathrm{Cl})$ \\
\hline \multirow[t]{5}{*}{ NS1 } & Brand A & $14.3 \%$ & $40.0 \%$ & $40.0 \%$ & $14.3 \%$ & $0.271(0.00-0.558)$ \\
\hline & Brand B & $7.1 \%$ & $100 \%$ & $100 \%$ & $27.8 \%$ & $0.536(0.243-0.828)$ \\
\hline & Brand C & $78.6 \%$ & $80.0 \%$ & $91.7 \%$ & $57.1 \%$ & $0.793(0.549-1.000)$ \\
\hline & Brand D & $21.4 \%$ & $80.0 \%$ & $75.0 \%$ & $26.7 \%$ & $0.507(0.206-0.808)$ \\
\hline & Brand $\mathrm{E}$ & $7.1 \%$ & $100 \%$ & $100 \%$ & $7.1 \%$ & $0.536(0.243-0.828)$ \\
\hline$E$ & Brand $\mathrm{F}^{\mathrm{a}}$ & $100 \%$ & $0 \%$ & $26.3 \%$ & $0 \%$ & $0.500(0.198-0.802)$ \\
\hline
\end{tabular}

aUnable to generate data for the parameters due to high level of positive values potentially caused by cross-reactivity. The results are not comparable. SS- sensitivity, SP - specificity, PPV - positive predictive value, NPV - Negative predictive value. AUC - area under the receiver operating characteristic curve.

and lastly the Brand A ELISA kit showed the specificity of $40.0 \%$.

As shown in Table 1, 14 samples were reported as ZIKV FRNT positives. Eight samples that were identified as ZIKV+ showed ZIKV FRNT 90 titer $\geq 1: 40$. In addition, six samples previously identified as positive to other viruses (JEV $=1$; $D E N V=4 ; C H I K V=1$ ) also presented $Z$ IKV FRNT 90 titer $\geq 1: 40$. The reasons for these are many and warrant further investigation.

\section{DISCUSSION}

In the present study, six commercial ELISA kits to detect ZIKV IgG antibodies were evaluated and compared to the reference FRNT. Five out of six ELISA kits employed NS1 as the antigen, whereas only the Brand F ELISA kit used the Eprotein corresponding to ZIKV epitopes. Our findings suggested that antibodies to $E$ protein were highly crossreactive between ZIKV, DENV, JEV, and CHIKV, whereas antibodies to the NS1 protein tended to be more virusspecific, this being which was in agreement with findings from previous studies (Stettler et al., 2016; Fritzell et al., 2018).

The sensitivity determined for the NS1-based ELISA varieds significantly from $7.1 \%$ to $78.6 \%$. Variations in sensitivity could be due to the unspecified ZIKV antigen used for each kit; low sensitivity could give false-negative results. These variations were also reported in several earlier studies, likely caused by the assay formats and detection system (Groen et al., 2000; Basile et al., 2018). In the present study, the Brand C ELISA kit had an overall sensitivity of $78.6 \%$ and specificity of $80.0 \%$ when compared against the FRNT. In an earlier study, the IgG kit was reported to have a high specificity $(94.2 \%)$ but a lower sensitivity of $34.4 \%$ (Huzly et al., 2016). Perhaps because the study only used symptomatic patients' acute samples for the evaluation. Other studies reported similar findings where the kits could be used with high background of pre-existing antibodies to other flaviviruses in endemic regions (Priyamvada et al., 2016). Notably, the Brand C ELISA kit had AUC values of 0.793 (95\% Cl: $0.549-1.000)$ which reflected its ability to differentiate true positive and true negative samples. On the other hand, while the specificity for both the Brand B and the Brand E ELISA kits reached $100 \%$, they had a very low sensitivity of $7.1 \%$. Although specificity is important to distinguish ZIKV from other viruses, sensitivity was crucial in determining the ELISA kit's usefulness. Confirmation with gold-standard FRNT can overcome false-positive results caused by specificity issues. However, false-negative results caused by poor sensitivity could miss the actual ZIKV positive sample and not be followed up for further testing.
The complexity of serological cross-reactivity in the detecting antibodies against flaviviruses is well documented (Fritzell et al., 2018; Paixão et al., 2018). The discrepancies between the ELISA tests and FRNT was observed among the validation serum panels with 19 sera in Phase II. Six samples previously identified as positive to other viruses were also positive to ZIKV FRNT. This may be due to a recent flavivirus infection that elicited cross-reactivity antibodies to ZIKV detected by ZIKV FRNT, causing false-positivity (Kikuti et al., 2018; Chao et al., 2019). Considering these 4 individuals could have had previous infection from the respective viruses, a 4-fold higher titer value against ZIKV as compared to other viruses should be expected. However, it is well reported that DENV-positive serum samples, especially those from secondary DENV infection, may cross-react with ZIKV (Dejnirattisai et al., 2016; Priyamvada et al., 2016; Swanstrom et al., 2016). Moreover, previous studies that performed 4fold higher titer value against ZIKV as compared to DENV could not conclude a specific cutoff value for the respective PRNT due to cross-reactivity (Netto et al., 2017; Schwarz et al., 2017; Mathé et al., 2018; Chien et al., 2019; Alves et al., 2020). The ZIKV and DENV PRNT titer could range from 1:10 to $1: 100$ 000 in secondary flavivirus infections (Lanciotti et al., 2008). In addition, the possibility of double infections, however, cannot be excluded as both infections could be present. It remains unclear if the ELISA seropositivity had resulted from the presence of ZIKV antibodies due to co-infection with ZIKV (true-positive) in the dengue infected patients' sera or from cross-reactivity (false-positive). Despite that, most of the evaluated ELISA kits in our study showed high specificity $(80.0-100 \%)$ using well-characterized, achieved serum panels, including ZIKV, DENV, JEV, and CHIKV, which are representative of populations in many tropical countries. It may represent a significant improvement on current commercially available ZIKV IgG diagnostic tools. Furthermore, since DENV is hyperendemic in Malaysia, both DENV and ZIKV circulate in the same geographic locations. Therefore, it is important to include serum samples from individuals with current DENV infection with prior exposure to ZIKV in future studies. Unfortunately, these types of serum collections are currently not available, but these samples may be included in the future as part of our research.

One major limitation of the present study was the small sample size of the Zika validation serum panels. Unfortunately, the serum collections were lacking due to the low presence of Zika infection among the rural Malaysian population where serum samples were obtained. Secondly, the serum panels were only validated by FRNT $_{90}(1: 40)$ against ZIKV without performing FRNT against DENV to 
differentiate serum panels that were reactive towards DENV from ZIKV. A two-tier FRNT study performed earlier showed that some samples cross-reacted with DENV (Sasmono et al., 2018). However, in the present study, we were unable to perform FRNT for all the viruses; it would have been be laborious, expensive, and would have used up a large amount of the serum samples. Therefore, establishing algorithm that only involve ELISAs to differentiate ZIKV infection from different DENV infections, especially secondary DENV infection, is urgently needed. This strategy is crucial for developing countries, where cell culture and PCR facilities are not widely available.

Notably, the present study still evaluated a large number of ZIKV serologic assays and contributed valuable information for their test performances in a dengue-endemic region. Based on the performance estimates reported here, using most NS1-based ELISA kits in dengue-endemic settings in screening would be expected to minimize false-positive results. This study highlighted the performance and technical experience using the ELISA kits for ZIKV detection. However, this would only be true for the serum panels used in our study. More follow-up studies using more samples would need to be done to verify if this suggestion remains acceptable.

\section{CONCLUSION}

Overall, the present study established that the performance of the NS1-based anti-ZIKV IgG ELISA kit was superior to that which uses of the $E$ protein as antigen. The performance of the NS1-based IgG ELISA, however, varies between the different kits. Cross-reactivity against other viruses especially DENV remains a challenge in the confirmation of previous Zika virus infection.

\section{ACKNOWLEDGEMENT}

This research was funded by Long Term Research Grant Scheme, LRGS MRUN/F1/01/2018 and Higher Institution Centre of Excellent (HICoE) program, MO002-2019 from the Ministry of Higher Education Malaysia.

\section{Conflict of interest}

The authors declare no competing conflicts of interest.

\section{REFERENCES}

Alves, L.V., Leal, C.A. \& Alves, J.G.B. (2020). Zika virus seroprevalence in women who gave birth during Zika virus outbreak in Brazil - a prospective observational study. Heliyon 6: e04817. https://doi.org/10.1016/j.heliyon. 2020.e04817

Basile, A.J., Goodman, C., Horiuchi, K., Sloan, A., Johnson, B.W., Kosoy, O., Laven, J., Panella, A.J., Sheets, I., Medina, F. et al. (2018). Multi-laboratory comparison of three commercially available Zika IgM enzyme-linked immunosorbent assays. Journal of Virological Methods 260: 26-33. https://doi.org/10.1016/j.jviromet.2018.06.018

Bossuyt, P.M., Reitsma, J.B., Bruns, D.E., Gatsonis, C.A., Glasziou, P.P., Irwig, L., Lijmer, J.G., Moher, D., Rennie, D., de Vet, H.C. et al. (2015). STARD 2015: an updated list of essential items for reporting diagnostic accuracy studies. British Medical Journal 351: h5527. https://doi.org/ 10.1136/bmj.h5527
Chao, D.Y., Whitney, M.T., Davis, B.S., Medina, F.A., Munoz, J.L. \& Chang, G.J. (2019). Comprehensive evaluation of differential serodiagnosis between Zika and dengue viral infections. Journal of Clinical Microbiology 57: e0150618. https://doi.org/10.1128/jcm.01506-18

Chien, Y.W., Ho, T.C., Huang, P.W., Ko, N.Y., Ko, W.C. \& Perng, G.C. (2019). Low seroprevalence of Zika virus infection among adults in Southern Taiwan. BMC Infectious Diseases 19: 884. https://doi.org/10.1186/s12879-019-4491-4

Dai, L., Song, J., Lu, X., Deng, Y.Q., Musyoki, A.M., Cheng, H., Zhang, Y., Yuan, Y., Song, H., Haywood, J. et al. (2016). Structures of the Zika virus envelope protein and its complex with a flavivirus broadly protective antibody. Cell Host \& Microbe 19: 696-704. https://doi.org/10.1016/ j.chom.2016.04.013

Dejnirattisai, W., Supasa, P., Wongwiwat, W., Rouvinski, A., Barba-Spaeth, G., Duangchinda, T., Sakuntabhai, A., CaoLormeau, V.M., Malasit, P., Rey, F.A. et al. (2016). Dengue virus sero-cross-reactivity drives antibody-dependent enhancement of infection with zika virus. Nature Immunology 17: 1102-1108. https://doi.org/10.1038/ni.3515

Dick, G.W., Kitchen, S.F. \& Haddow, A.J. (1952). Zika virus. I. Isolations and serological specificity. Transactions of the Royal Society Tropical Medicine and Hygiene 46: 509-520. https://doi.org/10.1016/0035-9203(52)90042-4

Fourcade, C., Mansuy, J.M., Dutertre, M., Delpech, M., Marchou, B., Delobel, P., Izopet, J. \& Martin-Blondel, G. (2016). Viral load kinetics of Zika virus in plasma, urine and saliva in a couple returning from Martinique, French West Indies. Journal of Clinical Virology 82: 1-4. https://doi.org/10.1016/ j.jcv.2016.06.011

Fritzell, C., Rousset, D., Adde, A., Kazanji, M., Van Kerkhove, M.D. \& Flamand, C. (2018). Current challenges and implications for dengue, chikungunya and Zika seroprevalence studies worldwide: A scoping review. PLOS Neglected Tropical Diseases 12: e0006533-e0006533. https://doi.org/10.1371/journal.pntd.0006533

Groen, J., Koraka, P., Velzing, J., Copra, C. \& Osterhaus, A.D. (2000). Evaluation of six immunoassays for detection of dengue virus-specific immunoglobulin $M$ and $G$ antibodies. Clinical and Diagnostic Laboratory Immunology 7: 867871. https://doi.org/10.1128/cdli.7.6.867-871.2000

Gutiérrez-Bugallo, G., Piedra, L.A., Rodriguez, M., Bisset, J.A., Lourenço-de-Oliveira, R., Weaver, S.C., Vasilakis, N. \& Vega-Rúa, A. (2019). Vector-borne transmission and evolution of Zika virus. Nature Ecology \& Evolution 3(4): 561-569. https://doi.org/10.1038/s41559-019-0836-z

Hajian-Tilaki, K. (2013). Receiver Operating Characteristic (ROC) Curve Analysis for Medical Diagnostic Test Evaluation. Caspian Journal of Internal Medicine 4: 627-635.

Huzly, D., Hanselmann, I., Schmidt-Chanasit, J. \& Panning, M. (2016). High specificity of a novel Zika virus ELISA in European patients after exposure to different flaviviruses. Eursurveillance 21: 30203. https://doi.org/ 10.2807/1560-7917.Es.2016.21.16.30203

Khor, C.S., Mohd-Rahim, N.F., Hassan, H., Tan, K.K., Zainal, N., Teoh, B.T., Sam, S.S., Khoo, J.J., Lee, H.Y., Lim, Y.A. \& Abubakar, S. (2020). Serological evidence of DENV, JEV, and ZIKV among the indigenous people (Orang Asli) of Peninsular Malaysia. Journal of Medical Virology 92: 956962. https://doi.org/10.1002/jmv.25649 
Kikuti, M., Tauro, L.B., Moreira, P.S.S., Campos, G.S., Paploski, I.A.D., Weaver, S.C., Reis, M.G., Kitron, U. \& Ribeiro, G.S. (2018). Diagnostic performance of commercial IgM and IgG enzyme-linked immunoassays (ELISAs) for diagnosis of Zika virus infection. Virology Journal 15: 108. https:// doi.org/10.1186/s12985-018-1015-6

Lanciotti, R.S., Kosoy, O.L., Laven, J.J., Velez, J.O., Lambert, A.J., Johnson, A.J., Stanfield, S.M. \& Duffy, M.R. (2008). Genetic and serologic properties of Zika virus associated with an epidemic, Yap State, Micronesia, 2007. Emerging Infectious Diseases 14: 1232. https://doi.org/10.3201/ eid1408.080287

Landry, M.L. \& St George, K. (2017). Laboratory diagnosis of Zika virus infection. Archives of Pathology \& Laboratory Medicine 141: 60-67. https://doi.org/10.5858/arpa.20160406-SA

Langerak, T., Brinkman, T., Mumtaz, N., Arron, G., Hermelijn, S., Baldewsingh, G., Wongsokarijo, M., Resida, L., Rockx, B., Koopmans, M.P.G. et al. (2019). Zika virus seroprevalence in urban and rural areas of Suriname, 2017. Journal of Infectious Diseases 220: 28-31. https://doi.org/10.1093/ infdis/jiz063

Lima, M., de Lima, R.C., de Azeredo, E.L. \& Dos Santos, F.B. (2021). Analysis of a routinely used commercial antichikungunya IgM ELISA reveals cross-reactivities with dengue in Brazil: A new challenge for differential diagnosis? Diagnostics (Basel) 11: 819. https://doi.org/ 10.3390/diagnostics11050819

Mathé, P., Egah, D.Z., Müller, J.A., Shehu, N.Y., Obishakin, E.T., Shwe, D.D., Pam, V.C., Okolo, M.O., Yilgwan, C., Gomerep, S.S. et al. (2018). Low Zika virus seroprevalence among pregnant women in North Central Nigeria, 2016. Journal of Clinical Virology 105: 35-40. https://doi.org/10.1016/ j.jcv.2018.05.011

Musso, D. \& Gubler, D.J. (2016). Zika Virus. Clinical Microbiology Reviews 29: 487-524. https://doi.org/10.1128/cmr.00072-15

Musso, D., Ko, A.I. \& Baud, D. (2019). Zika virus infection after the pandemic. New England Journal of Medicine 381: 1444-1457. https://doi.org/10.1056/NEJMra1808246

Netto, E.M., Moreira-Soto, A., Pedroso, C., Höser, C., Funk, S., Kucharski, A.J., Rockstroh, A., Kümmerer, B.M., Sampaio, G.S., Luz, E. et al. (2017). High Zika virus seroprevalence in Salvador, northeastern Brazil limits the potential for further outbreaks. MBio 8: e01390-17. https://doi.org/ 10.1128/mBio.01390-17

Nguyen, C.T., Moi, M.L., Le, T.Q.M., Nguyen, T.T.T., Vu, T.B.H., Nguyen, H.T., Pham, T.T.H., Le, T.H. T., Nguyen, L.M.H., Phu Ly, M.H. et al. (2020). Prevalence of Zika virus neutralizing antibodies in healthy adults in Vietnam during and after the Zika virus epidemic season: a longitudinal population-based survey. BMC Infectious Diseases 20: 332332. https://doi.org/10.1186/s12879-020-05042-2

Ohst, C., Saschenbrecker, S., Stiba, K., Steinhagen, K., Probst, C., Radzimski, C., Lattwein, E., Komorowski, L., Stöcker, W. \& Schlumberger, W. (2018). Reliable serological testing for the diagnosis of emerging infectious diseases. Advances in Experimental Medicine and Biology 1062: 19-43. https://doi.org/10.1007/978-981-10-8727-1_3

Paixão, E.S., Teixeira, M.G. \& Rodrigues, L.C. (2018). Zika, chikungunya and dengue: the causes and threats of new and re-emerging arboviral diseases. BMJ Global Health 3: e000530-e000530. https://doi.org/10.1136/bmjgh-2017000530
Priyamvada, L., Quicke, K.M., Hudson, W.H., Onlamoon, N., Sewatanon, J., Edupuganti, S., Pattanapanyasat, K., Chokephaibulkit, K., Mulligan, M.J., Wilson, P.C. et al. (2016). Human antibody responses after dengue virus infection are highly cross-reactive to Zika virus. Proceedings of the National Academy of Sciences 113: 7852. https://doi.org/10.1073/pnas.1607931113

Rabe, I.B., Staples, J.E., Villanueva, J., Hummel, K.B., Johnson, J.A., Rose, L., Hills, S., Wasley, A., Fischer, M. \& Powers, A.M. (2016). Interim guidance for interpretation of Zika virus antibody test results. MMWR Morbidity and Mortality Weekly Report 65: 543-546. https://doi.org/10.15585/ mmwr.mm6521e1

Roehrig, J.T., Hombach, J. \& Barrett, A.D.T. (2008). Guidelines for plaque-reduction neutralization testing of human antibodies to dengue viruses. Viral Immunology 21: 123132. https://doi.org/10.1089/vim.2008.0007

Saba Villarroel, P.M., Nurtop, E., Pastorino, B., Roca, Y., Drexler, J.F., Gallian, P., Jaenisch, T., Leparc-Goffart, I., Priet, S., Ninove, L. et al. (2018). Zika virus epidemiology in Bolivia: A seroprevalence study in volunteer blood donors. PLOS Neglected Tropical Diseases 12: e0006239-e0006239. https:/ /doi.org/10.1371/journal.pntd.0006239

Sam, I.C., Montoya, M., Chua, C.L., Chan, Y.F., Pastor, A. \& Harris, E. (2019). Low seroprevalence rates of Zika virus in Kuala Lumpur, Malaysia. Tranactions of the Royal Society of Tropical Medicine and Hygiene 113: 678-684. https://doi.org/10.1093/ trstmh/trz056

Sasmono, R.T., Dhenni, R., Yohan, B., Pronyk, P., Hadinegoro, S.R., Soepardi, E.J., Ma'roef, C.N., Satari, H.I., Menzies, H., Hawley, W.A. et al. (2018). Zika virus seropositivity in 1-4year-old children, Indonesia, 2014. Emerging Infectious Diseases 24: 1740-1743. https://doi.org/10.3201/eid2409. 180582

Schwarz, N.G., Mertens, E., Winter, D., Maiga-Ascofaré, O., Dekker, D., Jansen, S., Tappe, D., Randriamampionona, N., May, J., Rakotozandrindrainy, R. et al. (2017). No serological evidence for Zika virus infection and low specificity for anti-Zika virus ELISA in malaria positive individuals among pregnant women from Madagascar in 2010. PLOS ONE 12: e0176708. https://doi.org/10.1371/ journal.pone. 0176708

Song, H., Qi, J., Haywood, J., Shi, Y. \& Gao, G.F. (2016). Zika virus NS1 structure reveals diversity of electrostatic surfaces among flaviviruses. Nature Structural \& Molecular Biology 23: 456-458. https://doi.org/10.1038/nsmb.3213

Stettler, K., Beltramello, M., Espinosa, D.A., Graham, V., Cassotta, A., Bianchi, S., Vanzetta, F., Minola, A., Jaconi, S., Mele, F. et al. (2016). Specificity, cross-reactivity, and function of antibodies elicited by Zika virus infection. Science 353: 823-826. https://doi.org/10.1126/science. aaf8505

Swanstrom, J.A., Plante, J.A., Plante, K.S., Young, E.F., McGowan, E., Gallichotte, E.N., Widman, D.G., Heise, M.T., de Silva, A.M. \& Baric, R.S. (2016). Dengue virus envelope dimer epitope monoclonal antibodies isolated from dengue patients are protective against Zika virus. MBio 7: e0112316. https://doi.org/10.1128/mBio.01123-16

Vaidya, S.R., Brown, D.W., Jin, L., Samuel, D., Andrews, N. \& Brown, K.E. (2010). Development of a focus reduction neutralization test (FRNT) for detection of mumps virus neutralizing antibodies. Journal of Virology Methods 163: 153-156. https://doi.org/10.1016/j.jviromet.2009.09.006 
WHO. (2016a). Laboratory testing for Zika virus infection. World Health Organization. https://www.who.int/publications/ i/item/laboratory-testing-for-zika-virus-infection. Accessed 22 March 2020.

WHO. (2016b). Zika virus disease: interim case definition. World Health Organization. https://www.who.int/csr/ disease/zika/case-definition/en/. Accessed 13 April 2020.
WHO. (2019). Zika epidemiology update. World Health Organization. https://www.who.int/emergencies/diseases/ zika/zika-epidemiology-update-july-2019.pdf. Accessed 13 April 2020.

Wilder-Smith, A., Chang, C.R. \& Leong, W.Y. (2018). Zika in travellers 1947-2017: a systematic review. Journal of Travel Medicine 25. https://doi.org/10.1093/jtm/tay044

Supplementary files

Supplementary files available upon request. 